\section{A) Check for updates}

Cite this: Dalton Trans., 2018, 47, 5252

\title{
The impact of whole human blood on the kinetic inertness of platinum(Iv) prodrugs - an HPLC-ICP-MS study $\dagger$
}

\author{
Sarah Theiner, (D) a Márkó Grabarics, (DD b Luis Galvez, ${ }^{a}$ Hristo P. Varbanov, (D) c \\ Nadine S. Sommerfeld, ${ }^{\mathrm{C}}$ Mathea S. Galanski, ${ }^{\mathrm{C}}$ Bernhard K. Keppler ${ }^{\mathrm{c}, \mathrm{d}}$ and \\ Gunda Koellensperger*a
}

\begin{abstract}
The potential advantage of platinum(IV) complexes as alternatives to classical platinum(II)-based drugs relies on their kinetic stability in the body before reaching the tumor site and on their activation by reduction inside cancer cells. In this study, an analytical workflow has been developed to investigate the reductive biotransformation and kinetic inertness of platinum(Iv) prodrugs comprising different ligand coordination spheres (respectively, lipophilicity and redox behavior) in whole human blood. The distribution of platinum(Iv) complexes in blood pellets and plasma was determined by inductively coupled plasma-mass spectrometry (ICP-MS) after microwave digestion. An analytical approach based on reversed-phase (RP)-ICP-MS was used to monitor the parent compound and the formation of metabolites using two different extraction procedures. The ligand coordination sphere of the platinum(IV) complexes had a significant impact on their accumulation in red blood cells and on their degree of kinetic inertness in whole human blood. The most lipophilic platinum(IV) compound featuring equatorial chlorido ligands showed a pronounced penetration into blood cells and a rapid reductive biotransformation. In contrast, the more hydrophilic platinum(Iv) complexes with a carboplatin- and oxaliplatin-core exerted kinetic inertness on a pharmacologically relevant time scale with notable amounts of the compound accumulated in the plasma fraction.
\end{abstract}

\author{
Received 1st December 2017, \\ Accepted 27th February 2018 \\ DOI: $10.1039 / \mathrm{c} 7 \mathrm{dt} 04537 \mathrm{a}$ \\ rsc.li/dalton
}

\section{Introduction}

Platinum(Iv) complexes have the potential to overcome the shortcomings of established platinum(II)-based drugs (severe side effects, intravenous administration and acquired tumor resistance), due to their higher kinetic inertness to ligandexchange reactions. Based on the prodrug-concept, platinum(Iv) compounds are considered to reach the tumor site mainly in their intact form and to be activated to their platinum(II) analogues by reduction advantageously only inside cancer cells, resulting in less side effects and a lower toxicity profile and making them amenable for oral administration. ${ }^{1-3}$

\footnotetext{
${ }^{a}$ Institute of Analytical Chemistry, University of Vienna, Waehringer Strasse 38, 1090 Vienna, Austria. E-mail: gunda.koellensperger@univie.ac.at

${ }^{b}$ Department of Pharmacognosy, Semmelweis University, Üllöi út 26, 1085 Budapest, Hungary

${ }^{c}$ Institute of Inorganic Chemistry, University of Vienna, Waehringer Strasse 42, 1090 Vienna, Austria

${ }^{d}$ Research Cluster 'Translational Cancer Therapy Research', University of Vienna, Waehringer Strasse 42, 1090 Vienna, Austria

$\dagger$ Electronic supplementary information (ESI) available. See DOI: 10.1039/ c7dt04537a
}

However, most of our knowledge on the reduction of platinum(rv) prodrugs is based on experiments using simple model systems mimicking physiological conditions (e.g. ascorbate, cysteine, glutathione or other biological reducing agents in $\mathrm{PBS})^{4,5}$ Initial studies were focused on platinum(Iv) complexes, featuring a cisplatin-like core and indicated that the rate of reduction strongly depends on the nature of the axial ligands. Complexes with axial hydroxido ligands are the most resistant ones, agents bearing axial carboxylato ligands exhibit intermediate reduction rates, while those carrying axial chlorido ligands are the most readily and rapidly reduced platinum(Iv) compounds. ${ }^{6}$ More recently, also the significant role of equatorial ligands in the reduction rate of platinum(Iv) prodrugs has been shown: complexes with equatorial chlorido ligands are reduced more rapidly than their counterparts with carboxylato ligands. ${ }^{7-9}$ Despite these structure-activity relationships established in model systems (usually consisting of one reducing agent), little is known about the behavior of platinum(rv) compounds in complex biological fluids (e.g. in serum or blood) or in vivo. The incubation of platinum(Iv) compounds in aqueous extracts from different cancer cell lines demonstrated that high molecular weight biomolecules account for 
the majority of the reduction in cells and that the rate of reduction is dependent on the cell line used..$^{10,11}$ In the literature, some data are available regarding the in vivo metabolism of individual platinum(Iv) complexes that have undergone clinical trials (iproplatin, tetraplatin and satraplatin). ${ }^{12}$ In the case of satraplatin, seven platinum-containing species were already found in patient's plasma ultrafiltrate $15 \mathrm{~min}$ after oral administration whereas the parent compound could not be detected any more. ${ }^{13}$ Rapid biotransformation of satraplatin was also observed upon incubation with human red blood cells $^{14}$ and with hemoglobin and cytochrome $\mathrm{c}$ in the presence of NADH. ${ }^{15}$ Hambley et al. investigated the reactivity of platinum(Iv) complexes with different ligand coordination spheres towards selected plasma constituents (including human serum albumin and cysteine) and correlated the results with the reduction potentials. ${ }^{16}$

A variety of analytical techniques have been used to study platinum compounds and their interactions with proteins in blood compartments, including nuclear magnetic resonance (NMR) spectroscopy, electrospray ionization-mass spectrometry (ESI-MS) and atomic absorption spectroscopy (AAS). ${ }^{4,5}$ In recent years, hyphenated techniques combining chromatographic separation with sensitive and elementspecific detection techniques such as inductively coupled plasma-mass spectrometry (ICP-MS) have been effectively used to study the behavior of metallodrugs in biological systems. ${ }^{17}$ These studies addressed different aspects including e.g. the interaction of metallodrugs with serum proteins, ${ }^{18-21}$ protein binding kinetics, the reversibility of protein binding and the investigation of hydrolysis products and metabolites of metallodrugs $^{22}$ on a qualitative and/or quantitative basis. ${ }^{23-25}$

In this study, we present an analytical workflow based on extraction procedures and a RP-ICP-MS method to investigate the biotransformation of platinum(Iv) complexes in whole human blood. For this purpose, three hydrophilic complexes featuring a carboplatin- or oxaliplatin-core and two hydroxides or acetates on axial positions, together with one more lipophilic cisplatin analogue comprising two axial ethoxysuccinato ligands, were chosen for the study (Fig. 1). The impact of the coordination sphere on the penetration of the compounds into the blood cells and on their kinetic stability in whole blood was also evaluated.

\section{Experimental}

\section{Chemicals and reagents}

Ultrapure water (resistivity $\geq 18.2 \mathrm{M} \Omega \mathrm{cm}$ ) was obtained from a Milli-Q Element water purification system (Milli-Q Advantage, Darmstadt, Germany) and used for RP-ICP-MS experiments and for all dilutions for ICP-MS measurements. Nitric acid ( $\geq 69 \%$, p.a., TraceSelect, Fluka) was used for ICP-MS measurements. Elemental standard stock solutions of platinum and rhenium were obtained from CPI International (Amsterdam, The Netherlands). All other reagents and solvents were

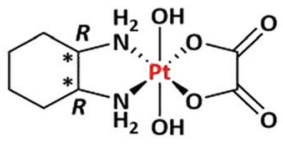

Complex 1

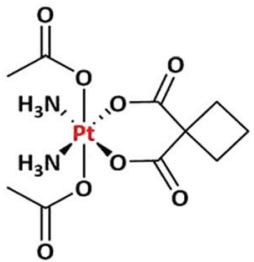

Complex 3

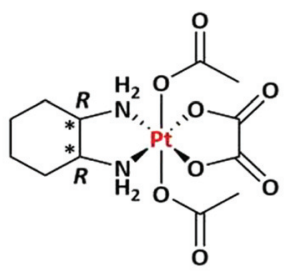

Complex 2
Fig. 1 Chemical formulae of the platinum(Iv) compounds under investigation.

obtained from commercial sources and were used without further purification.

Complex 1 ((OC-6-33)-dihydroxido(1R,2R-diaminocyclohexane)oxalatoplatinum(Iv)), complex 2 ((OC-6-33)-diacetato (1R,2R-diaminocyclohexane)oxalatoplatinum(Iv)) and complex 3 ((OC-6-33)-diacetatodiammine(1,1-cyclobutanedicarboxylato)platinum(Iv)) were synthesized according to literature procedures. $^{26,27}$ The synthesis and characterization of complex 4 ((OC-6-33)-dichloridobis((4-ethoxy)-4-oxobutanoato)bis(ethylamine) platinum(Iv)) are described in ref. 9 and 28. The chemical formulae of the platinum(Iv) complexes used in this study are shown in Fig. 1.

\section{Determination of the platinum concentration in blood compartments by ICP-MS}

Fresh human whole blood samples (collected in heparinized tubes), drawn in the framework of a biomarker study approved by the ethics committee of the Medical University of Vienna, were spiked ex vivo with an aqueous solution of the platinum(Iv) complexes $(1 \mathrm{mM})$ to reach a final concentration of $\sim 10 \mu \mathrm{M}$. The platinum concentrations were normalized to the hematocrit value of the whole blood (HCT $=47 \%$ ) and the density of plasma and blood pellets. Incubation was performed at $37^{\circ} \mathrm{C}$ for different time periods ( $0 \mathrm{~h}, 0.5 \mathrm{~h}, 1 \mathrm{~h}, 2 \mathrm{~h}$ and $24 \mathrm{~h}$ ). The separation of the blood plasma and blood cells was performed by centrifugation for $10 \mathrm{~min}$ at $2000 \mathrm{rcf}$, using a Hermle $\mathrm{Z}$ $446 \mathrm{~K}$ device (Hermle Labortechnik, Wehingen, Germany) with a pre-cooled rotor $\left(4^{\circ} \mathrm{C}\right)$. Clear fractions of the supernatant (plasma) and the pellet (blood cells) were stored at $-20{ }^{\circ} \mathrm{C}$ until further processing. Microwave-assisted acid digestion was performed by adding $2 \mathrm{~mL}$ of $25 \%$ nitric acid to a $50 \mu \mathrm{L}$ aliquot of each sample using a Discover SP-D (CEM Microwave Technology, Germany) microwave device operating with the following parameters: temperature $-200{ }^{\circ} \mathrm{C}$, ramp time - 
$4 \mathrm{~min}$, hold time - $6 \mathrm{~min}$, and maximal power - 300 W. The digested samples were further diluted with ultrapure water to achieve platinum levels in the working range of 0.1 to $20 \mu \mathrm{g} \mathrm{L}{ }^{-1}$. The determination of total platinum concentrations was performed by ICP-MS measurements, using an Agilent 7500ce quadrupole-based ICP-MS instrument (Agilent Technologies, Waldbronn, Germany). The ICP-MS parameters were tuned on a daily basis to achieve maximum sensitivity. The ICP-MS was equipped with a CETAC ASX-520 autosampler (Nebraska, USA), a MicroMist nebulizer with a sample uptake

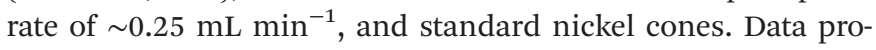
cessing was performed with the Agilent MassHunter software package (Workstation Software 4.3, Version B.01.01, 2012). The instrumental parameters of the ICP-MS measurements are summarized in Table S1. $\dagger$

\section{Extraction of platinum(Iv) compounds from whole blood using} cold organic extraction (Method A)

The spiking of fresh human blood (collected in heparinized tubes) was performed by adding $10 \mu \mathrm{L}$ of $100 \mu \mathrm{M}$ aqueous solution of platinum(Iv) compounds to $90 \mu \mathrm{L}$ of fresh whole human blood. $900 \mu \mathrm{L}$ ice-cold acetonitrile was added to the spiked blood samples to disrupt cells and precipitate proteins, followed by $15 \mathrm{~s}$ of vortexing. The samples were shaken for $30 \mathrm{~s}$, sonicated for $1 \mathrm{~min}$ and again vortexed for $15 \mathrm{~s}$. Finally, the samples were centrifuged for $1 \mathrm{~min}$ at $14000 \mathrm{rpm}$ using a Hettich Mikro 120 device (Hettich Zentrifugen, Tuttlingen, Germany). The supernatants were filtered directly into HPLC vials, using Sartorius Minisart syringe filters with a pore size of $0.45 \mu \mathrm{m}$ and diluted in the ratio of $1: 5$ with $50 \mathrm{mM}$ ammonium-formate buffer $(\mathrm{pH}=6.0)$. The vials were placed into the pre-cooled autosampler $\left(4^{\circ} \mathrm{C}\right)$ of the HPLC system for RP-ICP-MS analysis. In order to determine the extraction recovery, the weight of the filtered supernatants was measured and a $500 \mu \mathrm{L}$ aliquot of each filtrate was weighed, followed by the

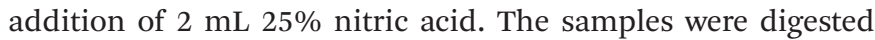
using a Discover SP-D (CEM Microwave Technology, Germany) microwave device with the following parameters: temperature $200{ }^{\circ} \mathrm{C}$, ramp time $-4 \mathrm{~min}$, hold time $-6 \mathrm{~min}$, and maximal power $-300 \mathrm{~W}$. The platinum concentration of the samples was measured by ICP-MS, using the same conditions as described above with the instrumental parameters summarized in Table S1. $\dagger$

\section{Extraction of platinum(Iv) compounds from whole blood with} acidic extraction and freeze-thawing (Method B)

The spiking was performed by adding $20 \mu \mathrm{L}$ of $100 \mu \mathrm{M}$ aqueous drug solution to $180 \mu \mathrm{L}$ fresh whole human blood and $200 \mu \mathrm{L}$ of $0.1 \% \mathrm{HCOOH}$ was added to the spiked blood samples, followed by vortexing for $15 \mathrm{~s}$. The samples were transferred onto dry-ice for $1 \mathrm{~min}$ (freezing) and then sonicated for 1 min using a water bath of $20{ }^{\circ} \mathrm{C}$ (thawing). The samples were vortexed for $15 \mathrm{~s}$ and centrifuged for $1 \mathrm{~min}$ at 14000 rpm using a Hettich Mikro 120 device (Hettich Zentrifugen, Tuttlingen, Germany) to verify the completeness of the cell lysis. After the first centrifugation step, 25\% (w/v) trichloroacetic acid was added to each sample to cause protein precipitation, followed by $15 \mathrm{~s}$ of vortexing, sonication for $1 \mathrm{~min}$ and another vortex step for $15 \mathrm{~s}$. Centrifugation was performed for a second time under the same conditions as described above. The supernatants were filtered using Sartorius Minisart syringe filters with a pore size of $0.45 \mu \mathrm{m}$ and then diluted in the ratio of $1: 5$ with $50 \mathrm{mM}$ ammoniumformate buffer $(\mathrm{pH}=6.0)$ directly into HPLC vials. The vials were placed into the pre-cooled autosampler $\left(4^{\circ} \mathrm{C}\right)$ of the HPLC system for RP-ICP-MS analysis. In order to determine the extraction efficiency, the supernatants obtained after the second centrifugation step were filtered, weighted and diluted with $3 \%$ nitric acid. The platinum concentration of the samples was determined by ICP-MS, using the same measurement conditions as described above and the instrumental parameters as summarized in Table S1.†

\section{Monitoring the biotransformation of platinum(Iv) complexes by RP-ICP-MS}

RP-ICP-MS measurements were performed on an Agilent 1260 Infinity Bio-inert HPLC system (Agilent Technologies, Waldbronn, Germany) hyphenated to an Agilent 8800 ICP-MS/ MS instrument (Agilent Technologies, Tokyo, Japan). Chromatographic separations were performed using an Atlantis T3 C18 analytical column $(150 \mathrm{~mm} \mathrm{~L} \times 2.1 \mathrm{~mm}$ i.d., $3 \mu \mathrm{m} \mathrm{d}_{\mathrm{p}}, 100 \AA$ pore size) equipped with an Atlantis T3 guard column $\left(20 \mathrm{~mm} \mathrm{~L} \times 2.1 \mathrm{~mm}\right.$ i.d., $\left.3 \mu \mathrm{m} \mathrm{d}_{\mathrm{p}}\right)$ from Waters (Milford, USA). Chromatographic conditions are summarized in Table S2. $\uparrow$ The ICP-MS/MS was equipped with a torch with $1.5 \mathrm{~mm}$ inner diameter, platinum cones and the brass-based lens system in order to withstand the high organic solvent load. Oxygen was introduced as the option gas and admixed with the aerosol before entering the ICP-MS to avoid carbon deposition on the cones. Data processing was performed with the Agilent MassHunter software package (Workstation Software, Version C.01.03, 2016). The ICP-MS operation parameters and chromatographic conditions used are summarized in Tables S1 and S2, $\uparrow$ respectively. Samples were measured in triplicates and an aqueous solution of complex $3(50 \mu \mathrm{M})$ was measured after every third sample as quality control.

\section{Results and discussion}

\section{Determination of the platinum concentration in blood compartments by ICP-MS}

The platinum concentration of the compounds under investigation was determined by ICP-MS in whole blood, blood pellets and plasma after centrifugation and microwave-assisted acid digestion at different time points over 24 hours (Fig. 2). Complexes 1-3 showed a similar platinum distribution pattern in blood compartments. Initially, the three platinum(Iv) compounds were predominantly localized in the plasma ( $\sim 80-90 \%$ of platinum). After $24 \mathrm{~h}, 20-30 \%$ of platinum was found in the blood cells, indicating a slow penetration of the compounds and/or their metabolites from the plasma into the blood cells. 

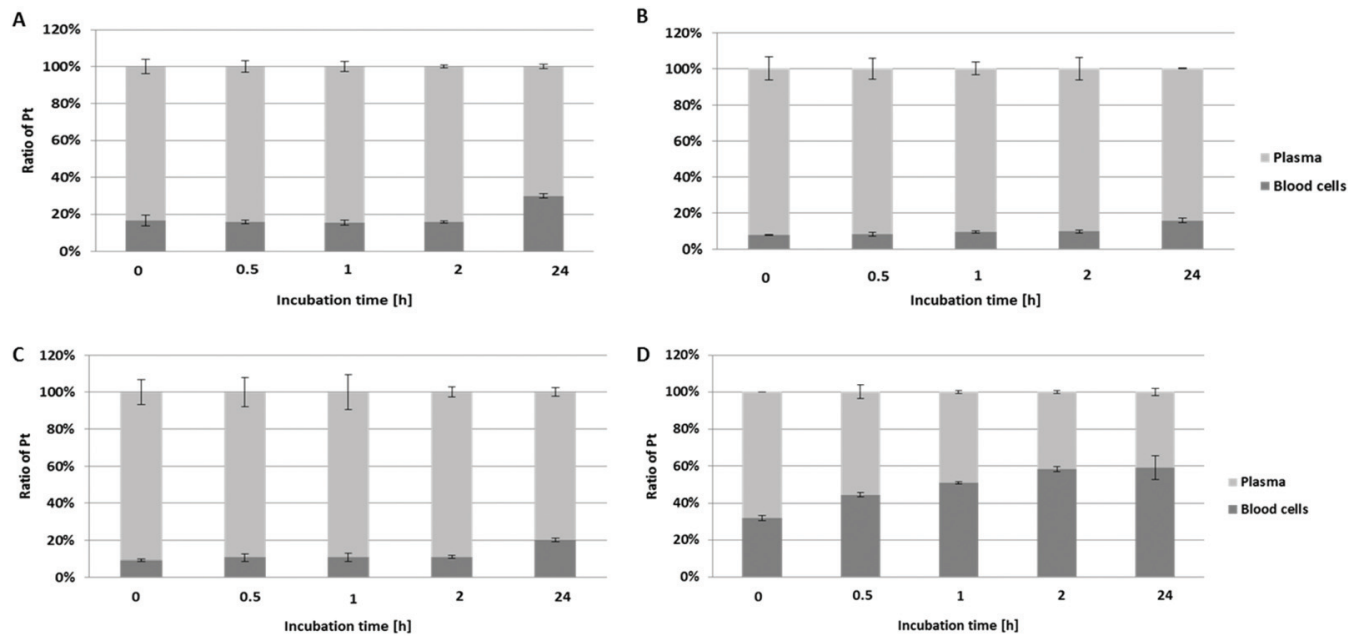

Fig. 2 Distribution of drug-related platinum in plasma and blood cells upon incubation of four different platinum(IV) compounds in whole blood. A: complex 1, B: complex 2, C: complex 3, and D: complex 4. Blood samples were spiked with pharmacologically relevant concentrations of the platinum(Iv) compounds $(10 \mu \mathrm{M})$ and platinum concentrations were determined in different blood fractions by ICP-MS after centrifugation and microwave digestion. Error bars correspond to the standard deviation of three methodological replicates.

However, there was no significant change observed in the platinum distribution of the compounds after $2 \mathrm{~h}$, compared to time point 0 . These results could be explained by the hydrophilic character expressed as negative $\log P_{\mathrm{o} / \mathrm{w}}$ values of the complexes (summarized in Table 1), which might prevent them from passing through biological membranes efficiently. Complex $\mathbf{4}$, in contrast, accumulates $30 \%$ of platinum already at time point 0 in the red blood cells. It also penetrates the blood cells in a significantly faster way than the other three platinum(Iv) complexes investigated, with around $60 \%$ of platinum found in blood cells after $2 \mathrm{~h}$. One possible explanation of these phenomena might be the higher lipophilicity of complex $4\left(\log P_{\mathrm{o} / \mathrm{w}}=0.78\right.$, Table 1) compared to the other investigated platinum(Iv) compounds. Complex 4 also displayed rapid saturation kinetics of accumulation: the distribution of drug-related platinum after 2 hours is close to that observed after 24 hours of incubation. Assuming exponential kinetics, the rate constant of the accumulation of complex 4 in blood cells was calculated from the proportion of the drugrelated platinum in the cells as a function of time. According

Table 1 Extraction efficiency of the platinum(iv) complexes from whole human blood using extraction method $A$ with acetonitrile and extraction method B with freeze-thawing. The values are given as the average value and standard deviation from three independent methodological replicates. The lipophilicity of the compounds is expressed as the octanol-water partition coefficient $\left(\log P_{\mathrm{o}} / \mathrm{w}\right)$, calculated by employing a model specially developed for the prediction of $\log P$ values of platinum (II) and platinum(IV) complexes ${ }^{30}$

\begin{tabular}{lllc}
\hline & $\begin{array}{l}\text { Recovery (\%), } \\
\text { Method A }\end{array}$ & $\begin{array}{l}\text { Recovery (\%), } \\
\text { Method B }\end{array}$ & $\log P_{\mathrm{o} / \mathrm{w}}$ \\
\hline Complex 1 & $11.7 \pm 1.1$ & $75.7 \pm 2.3$ & -2.1 \\
Complex 2 & $60.4 \pm 1.3$ & $75.9 \pm 1.2$ & -1.4 \\
Complex 3 & $60.5 \pm 2.3$ & $79.8 \pm 1.2$ & -1.5 \\
Complex 4 & $73.4 \pm 4.3$ & $40.3 \pm 0.8$ & 0.78
\end{tabular}

to the kinetic model, $59.7 \pm 0.9 \%$ of the drug-related platinum can be found inside the blood cells in equilibrium, with a first-order rate constant of $1.2 \pm 0.1 \mathrm{~h}^{-1}$. These results are in good agreement with a previous in vivo study addressing the anticancer efficacy of complex 4 in a murine CT-26 colon cancer model and its platinum distribution in different mice organs, tumors, blood pellets and serum..$^{29}$ In this case, $24 \mathrm{~h}$ after the last oral administration (either after single or multiple administrations of compound 4 ) around $60 \%$ of platinum was found in the blood pellet of the mice. ${ }^{29}$ Recent in vitro studies also revealed high platinum accumulation in SW480 (colon carcinoma) cells after $2 \mathrm{~h}$ exposure to complex $4 .{ }^{9}$

\section{Evaluation of extraction procedures for platinum(Iv) compounds from whole human blood}

Investigating the kinetic inertness of platinum(Iv) complexes in whole human blood requires a rapid and robust sample preparation. For this purpose, two different sample preparation protocols have been evaluated to extract platinum(Iv) complexes directly from whole human blood with respect to their extraction efficiency and time consumption. Method A was based on ice-cold acetonitrile for extraction and the extraction efficiency values are summarized in Table 1. For complexes 2 and 3, extraction efficiencies of approx. $60 \%$ were obtained, whereas compound $\mathbf{4}$ was extracted to an extent of $\sim 75 \%$. However, the procedure turned out to be unsuitable for the most hydrophilic compound $\mathbf{1}$, showing a poor recovery between 10 and $15 \%$ which could be also due to the poor solubility of complex $\mathbf{1}$ in acetonitrile. For this reason, a second extraction method was developed and evaluated using freezethawing for cell disruption and trichloroacetic acid for protein precipitation, avoiding the usage of organic solvents (Method $\mathrm{B}$, extraction efficiency values are summarized in Table 1). This method yielded higher extraction efficiencies for all platinum(Iv) 
complexes (between 70 and 80\%) compared to method A, except for the most lipophilic compound 4, which was only extracted to an extent of around $40 \%$. The advantages of method A are its simplicity and low time requirement (less than 4 minutes). Furthermore, this method is directly compatible with hydrophilic interaction liquid chromatography as acetonitrile serves as the weak eluent in the case of this chromatographic mode. As a consequence, time-consuming evaporation and solvent reconstitution can be avoided. Extraction method B includes more extraction steps and is more timeconsuming (approx. 9 minutes) than method A. The extraction efficiency is comparable or higher than for method A except for compound 4. The purely aqueous extracts obtained with this method can be directly injected into a reversed-phase column. Based on these results, the cold organic extraction method (method A) was used for complex $\mathbf{4}$ and the acidic extraction method (method B) for the other three platinum(Iv) compounds to directly extract the complexes and their metabolites from whole human blood.

\section{Studying the biotransformation of platinum(Iv) complexes in whole human blood using RP-ICP-MS}

The platinum(Iv) compounds were incubated in whole human blood over $24 \mathrm{~h}$ and the developed extraction methods were used to monitor their kinetics of biotransformation in whole human blood by RP-ICP-MS. Overlaid chromatograms of platinum(Iv) compounds are shown in Fig. 3. The results indicate that complex 1 and 3 remain stable over 24 hours in blood, as no metabolites or decrease of the signal corresponding to the parent compound was observed in the chromatograms. In the case of complex 2, only the intact complex was observed over a time period of 6 hours. After one day, the parent compound and also a significant amount of oxaliplatin could be detected, most likely due to the reductive biotransformation in the blood. However, as every xenobiotic agent in the bloodstream would travel through the liver (and other organs with metabolic capacity) multiple times during the 6 hour-period, complex 2 can also be regarded stable in blood on a pharmacologically relevant timescale. Compound $\mathbf{4}$, in contrast, showed rapid metabolisation and a decrease of the signal corresponding to the parent compound in the blood; the intact prodrug could not be detected any more after 24 hours of incubation. The half-life of complex 4 in blood was calculated to be $45.4 \pm 3.3 \mathrm{~min}$, which was determined by fitting a first-order kinetic model to the data illustrated in Fig. 3. There seems to be a correlation observable between the extent of penetration into blood cells (Fig. 2) and the kinetics of biotransformation of the platinum(Iv) compounds. Complexes 1-3 remain mostly in the plasma, resulting also in a comparable stability towards biotransformation on a pharmacologically relevant time scale. Compound 4, in contrast, accumulates platinum to a higher extent in blood cells ( $30 \%$ at $0 \mathrm{~h}$ and $60 \%$ at $2 \mathrm{~h}$ ) resulting in a short half-life in blood, which clearly demonstrates the important role of blood cells in the metabolism of platinum(Iv) complexes. Furthermore, no notable amounts of hydrophilic platinum(II)-metabolites were detected for 4 after $24 \mathrm{~h}$ in blood, while in the case of 2 , a significant amount of free, unbound, extractable oxaliplatin could be observed after the same incu-
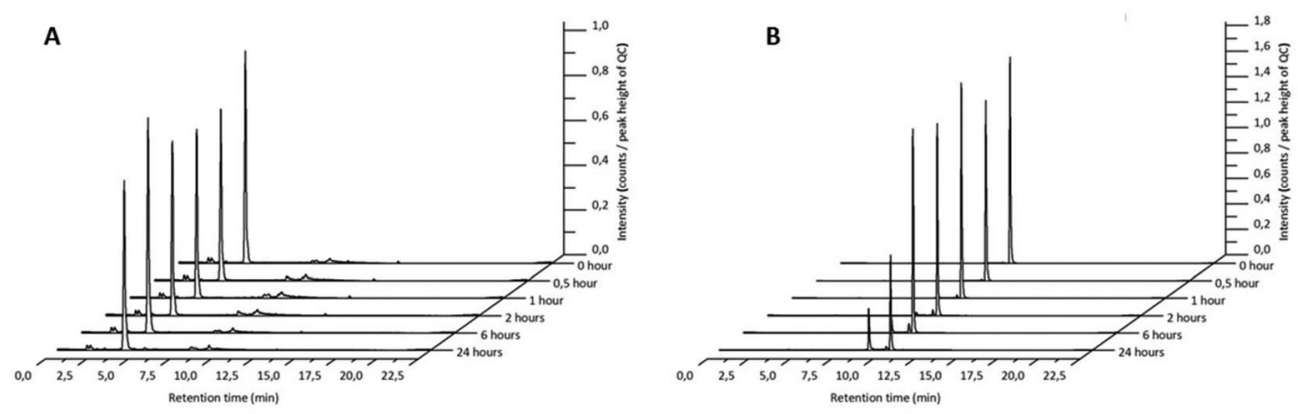

C

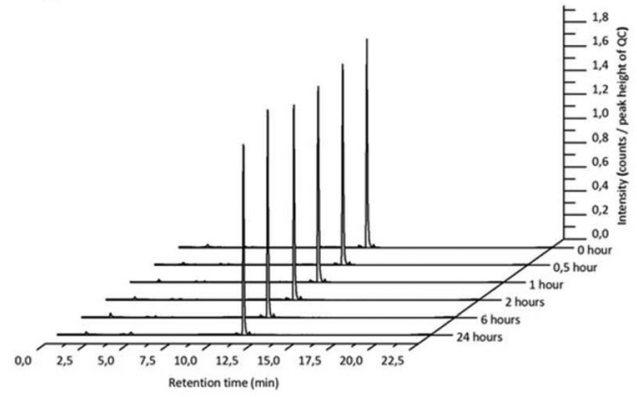

D

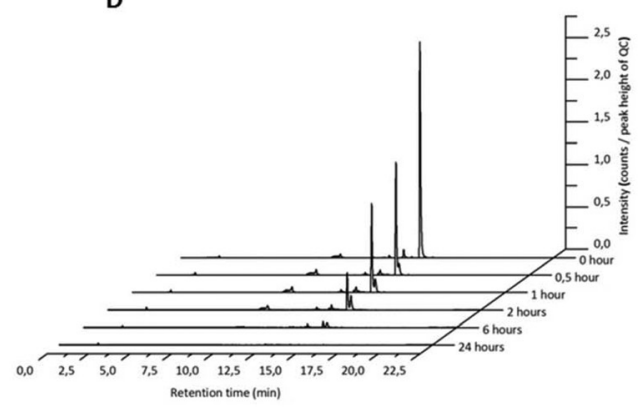

Fig. 3 RP-ICP-MS chromatograms of the platinum(IV) compounds after incubation $(10 \mu \mathrm{M})$ in whole human blood for $0 \mathrm{~h}, 0.5 \mathrm{~h}, 1 \mathrm{~h}, 2 \mathrm{~h}, 6 \mathrm{~h}$ and $24 \mathrm{~h}$ at $37^{\circ} \mathrm{C}$. (A) Complex 1, (B) complex 2, (C) complex 3 and (D) complex 4. Samples were measured in triplicates and an aqueous solution of complex 3 was injected after every third sample as quality control and used for normalisation. 
bation period. One reason behind these phenomena might be the more reactive platinum(II)-core of complex 4. ${ }^{9,28}$ Compound $\mathbf{4}$ is featuring fast-leaving equatorial chlorido ligands, which are capable of binding covalently to proteins with much faster kinetics compared to the oxaliplatin- or carboplatin-core. However, other reasons might also be the higher lipophilicity and the faster reduction of compound 4 compared to complexes 1-3. The biotransformation of complex 4 is in accordance with the results obtained for satraplatin during evaluation in clinical trials, which showed rapid metabolisation once in the bloodstream with several metabolites found in plasma ultrafiltrate of patients. ${ }^{13}$ Incubation of satraplatin in whole human blood in vitro resulted in a halflife for the disappearance of the parent compound of only $6.3 \mathrm{~min}$ which the authors attributed to the presence of red blood cells. ${ }^{14}$ Despite the observed rapid metabolism of satraplatin in the bloodstream, it advanced to clinical phase III trials, ${ }^{12,31}$ which suggests that platinum(Iv) complexes are sufficiently stable to be used as oral drugs. Also in the case of complex 4, despite the extensive biotransformation observed in this study, it showed anticancer activity in a murine colon cancer model CT-26 after i.p. and p.o. administration as well as efficacy in the leukemia L1210 mouse model. ${ }^{29}$

\section{Conclusions}

The fate of platinum(Iv) compounds comprising different coordination spheres was investigated in whole human blood by studying (1) the fractionation of the drugs between blood cells and plasma next to (2) the chemical inertness of the intact and free platinum(Iv) compounds upon ex vivo incubation. The latter task could be fulfilled after developing fit for purpose extraction protocols followed by RP-ICP-MS analysis.

The study showed that the extent of biotransformation was dependent on the platinum accumulation in blood cells, indicating that the environment plays an important role in the reduction of platinum(Iv) agents. Moreover, the coordination sphere also seems to have an impact on the reduction of platinum(Iv) complexes in blood, as the most significant biotransformation was observed for the platinum(rv) complex bearing equatorial chlorido ligands.

However, careful balancing between inertness and reactivity and between selectivity and cytotoxicity cannot be emphasized enough: if platinum(Iv) agents reach their targets successfully, but are not reduced and do not release cytotoxic platinum(II) agents, their anticancer activity in vivo would be unsatisfactory. Therefore, further studies are needed to find the optimal coordination spheres with the desired rate of reduction, besides investigating the effect of the liver and other organs on the biotransformation of platinum(rv) agents.

\section{Conflicts of interest}

There are no conflicts of interest to declare.

\section{Acknowledgements}

H. V. is thankful for the financial support from the Austrian Science Fund (FWF, Schrödinger fellowship J3577-B13).

\section{References}

1 M. D. Hall and T. W. Hambley, Coord. Chem. Rev., 2002, 232, 49-67.

2 T. C. Johnstone, K. Suntharalingam and S. J. Lippard, Chem. Rev., 2016, 116, 3436-3486.

3 D. M. Cheff and M. D. Hall, J. Med. Chem., 2017, 60, 45174532.

4 D. Gibson, Dalton Trans., 2016, 45, 12983-12991.

5 E. Wexselblatt and D. Gibson, J. Inorg. Biochem., 2012, 117, 220-229.

6 S. Choi, C. Filotto, M. Bisanzo, S. Delaney, D. Lagasee, J. L. Whitworth, A. Jusko, C. Li, N. A. Wood, J. Willingham, A. Schwenker and K. Spaulding, Inorg. Chem., 1998, 37, 2500-2504.

7 C. K. J. Chen, J. Z. Zhang, J. B. Aitken and T. W. Hambley, J. Med. Chem., 2013, 56, 8757-8764.

8 D. Höfer, H. P. Varbanov, M. Hejl, M. A. Jakupec, A. Roller, M. Galanski and B. K. Keppler, J. Inorg. Biochem., 2017, 174, 119-129.

9 S. Göschl, H. P. Varbanov, S. Theiner, M. A. Jakupec, M. Galanski and B. K. Keppler, J. Inorg. Biochem., 2016, 160, 264-274.

10 A. Nemirovski, Y. Kasherman, Y. Tzaraf and D. Gibson, J. Med. Chem., 2007, 50, 5554-5556.

11 J. Banfic, M. S. Adib-Razavi, M. Galanski and B. K. Keppler, Z. Anorg. Allg. Chem., 2013, 639, 1613-1620.

12 N. J. Wheate, S. Walker, G. E. Craig and R. Oun, Dalton Trans., 2010, 39, 8113-8127.

13 F. I. Raynaud, P. Mistry, A. Donaghue, G. K. Poon, L. R. Kelland, C. F. J. Barnard, B. A. Murrer and K. R. Harrap, Cancer Chemother. Pharmacol., 1996, 38, 155-162.

14 J. L. Carr, M. D. Tingle and M. J. McKeage, Cancer Chemother. Pharmacol., 2002, 50, 9-15.

15 J. Carr, M. Tingle and M. McKeage, Cancer Chemother. Pharmacol., 2006, 57, 483-490.

16 R. C. Dolman, G. B. Deacon and T. W. Hambley, J. Inorg. Biochem., 2002, 88, 260-267.

17 B. Gammelgaard, H. R. Hansen, S. Stürup and C. Møller, Expert Opin. Drug Metab. Toxicol., 2008, 4, 1187-1207.

18 G. Koellensperger, M. Galanski, B. K. Keppler and S. Hann, J. Anal. At. Spectrom., 2016, 31, 1811-1817.

19 A. K. Bytzek, K. Boeck, G. Hermann, S. Hann, B. K. Keppler, C. G. Hartinger and G. Koellensperger, Metallomics, 2011, 3, 1049-1055.

20 M. Groessl, M. Terenghi, A. Casini, L. Elviri, R. Lobinski and P. J. Dyson, J. Anal. At. Spectrom., 2010, 25, 305-313.

21 N. S. Sommerfeld, D. Strohhofer, K. Cseh, S. Theiner, M. A. Jakupec, G. Koellensperger, M. Galanski and B. K. Keppler, Eur. J. Inorg. Chem., 2017, 2017, 4049-4054. 
22 T. Falta, G. Koellensperger, A. Standler, W. Buchberger, R. M. Mader and S. Hann, J. Anal. At. Spectrom., 2009, 24, 1336-1342.

23 M. Groessl and C. Hartinger, Anal. Bioanal. Chem., 2013, 405, 1791-1808.

24 B. Gammelgaard, S. Stürup, C. Møller and A. V. Lyubimov, in Encyclopedia of Drug Metabolism and Interactions, John Wiley \& Sons, Inc., 2011.

25 S. Theiner, L. Galvez-Montano, G. Koellensperger and B. K. Keppler, in Analytical Techniques and Speciation Methods, ed. B. Michalke, Wiley-VCH, Weinheim, Germany, 2015.

26 D. Höfer, H. P. Varbanov, A. Legin, M. A. Jakupec, A. Roller, M. Galanski and B. K. Keppler, J. Inorg. Biochem., 2015, 153, 259-271.

27 J. Banfić, A. A. Legin, M. A. Jakupec, M. Galanski and B. K. Keppler, Eur. J. Inorg. Chem., 2014, 2014, 484-492.
28 H. Varbanov, S. M. Valiahdi, A. A. Legin, M. A. Jakupec, A. Roller, M. Galanski and B. K. Keppler, Eur. J. Med. Chem., 2011, 46, 54565464.

29 S. Theiner, H. Varbanov, M. Galanksi, A. Egger, W. Berger, P. Heffeter and B. K. Keppler, J. Biol. Inorg. Chem., 2015, 20, 89-99.

30 I. V. Tetko, H. P. Varbanov, M. Galanski, M. Talmaciu, J. A. Platts, M. Ravera and E. Gabano, J. Inorg. Biochem., 2016, 156, 1-13.

31 C. N. Sternberg, D. P. Petrylak, O. Sartor, J. A. Witjes, T. Demkow, J. M. Ferrero, J. C. Eymard, S. Falcon, F. Calabro, N. James, I. Bodrogi, P. Harper, M. Wirth, W. Berrry, M. E. Petrone, T. J. McKearn, M. Noursalehi, M. George and M. Rozencweig, J. Clin. Oncol., 2009, 27, 5431-5438. 